\title{
Charging Symmetries and Linearizing Potentials for Heterotic String in Three Dimensions
}

\author{
Alfredo Herrera-Aguilar \\ Joint Institute for Nuclear Research, \\ Dubna, Moscow Region 141980, RUSSIA. \\ e-mail: alfa@cv.jinr.dubna.su \\ and \\ Oleg Kechkin \\ Institute of Nuclear Physics, \\ Moscow State University, \\ Moscow 119899, RUSSIA, \\ e-mail: kechkin@monet.npi.msu.su
}

\begin{abstract}
Using the Ernst potential formulation we construct all finite symmetry transformations which preserve asymptotics of the bosonic fields of the $(d+3)$-dimensional low-energy heterotic string theory compactified on a $d$-torus. We combine all the dynamical variables into a single $(d+1) \times(d+1+n)$-dimensional matrix potential which linearly transforms under the action of these symmetry transformations in a transparent $S O(2, d-1) \times S O(2, d-1+n)$ way, where $\mathrm{n}$ is the number of Abelian vector fields. We formulate the most general solution generation technique based on the use of these symmetries and show that they form an invariance group of the general Israel-Wilson-Perj'es class of solutions.
\end{abstract}




\section{Introduction}

There are some strong arguments that superstring theory provides a correct quantum description of all fundamental forces including gravity [1]. In the low-energy limit five $D=10$ consistent perturbative superstring theories, as well as eleven-dimensional supergravity, lead to some modifications of General Relativity; these theories are interrelated by a web of superstring dualities, giving rise to M-theory [2]. Usually one considers the bosonic sector of these superstring gravity models and extracts from this sector supersymmetric solutions, i.e. the on-shell bosonic fields which do not generate any superfields under the action of supersymmetry transformations. These supersymmetric, or BPS-saturated solutions, do not obtain quantum corrections and form a basic tool for the study of the non-perturbative aspects of superstring theory [3].

In this paper we develop new formalism for construction and symmetry analysis of the bosonic solutions to the gravity model arising in the framework of heterotic string (HS) theory. In $D$ dimensions, its effective action reads:

$$
S^{(D)}=\int d^{(D)} x\left|G^{(D)}\right|^{\frac{1}{2}} e^{-\phi^{(D)}}\left(R^{(D)}+\phi_{; M}^{(D)} \phi^{(D) ; M}-\frac{1}{12} H_{M N P}^{(D)} H^{(D) M N P}-\frac{1}{4} F_{M N}^{(D) I} F^{(D) I M N}\right),
$$

where

$F_{M N}^{(D) I}=\partial_{M} A_{N}^{(D) I}-\partial_{N} A_{M}^{(D) I}, \quad H_{M N P}^{(D)}=\partial_{M} B_{N P}^{(D)}-\frac{1}{2} A_{M}^{(D) I} F_{N P}^{(D) I}+$ cycl perms of $\quad \mathrm{M}, \mathrm{N}, \mathrm{P}$.

Here $G_{M N}^{(D)}$ is the metric, $B_{M N}^{(D)}$ is the anti-symmetric Kalb-Ramond field, $\phi^{(D)}$ is the dilaton and $A_{M}^{(D) I}$ is the set of $U(1)$ vector fields $(I=1,2, \ldots, n)$. In the consistent critical case $D=10$ and $n=16$, but we shall leave these parameters arbitrary in our analysis. Following Maharana, Schwarz [4] and Sen [5], we consider the compactification of this model on a $D-3=d$-torus. The resulting three-dimensional theory possesses the $S O(d+1, d+1+n)$ symmetry group [5] (U-duality [6]).

Below we separate U-duality to the gauge and non-gauge sectors. Next, we fix the gauge (the trivial field asymptotics) and construct a representation of the theory which linearizes the non-gauge sector. Transformations of this sector form a charging symmetry (CS) subgroup. They generate charged solutions from neutral ones (see [7] for CS in the Einstein-Maxwell (EM) theory).

This paper is organized as follows. In Sec. 2 we review the matrix Ernst potential (MEP) formulation for HS in three dimensions [8]. We show how the EM theory in its ordinary (complex) Ernst potential formulation [9] arises as some very special case of the HS theory in the framework of MEP approach. In Sec. 3 we obtain all the CS transformations 
in a finite form using the MEP formulation. After that in Sec. 4 we introduce a pair of new matrix variables and show that they transform linearly under the action of the all CS transformations. We derive these linearizing potentials (LP) for the EM theory using the complex Ernst potential formulation (the details can be found in the Appendix A), and directly generalize the result to the general HS theory case.

Next, we combine both LP into a single $(d+1) \times(d+1+n)$ linearizing potential and show that it transforms in a manifest $S O(2, d-1) \times S O(2, d-1+n)$ form. This allow us to establish the CS group structure (the non-trivial structure of its $s o(2, d-1+n)$ subalgebra is studied in the Appendix B). After that we construct from this single LP one charging symmetry invariant (CSI) which is closely related to the charge quadratic function vanishing for the BPS-saturated fields.

Sec. 5 contains an investigation of the HS fields with a linear dependence between the linearizing potentials. First, we show that such fields are invariant under the action of charging symmetries, so that this class of fields cannot be generalized using the CS transformations. By setting the CSI to zero we get a restriction on this class. The corresponding subclass coincides with the general class of Israel-Wilson-Perj'es (IWP) solutions of the three-dimensional heterotic string theory. We show that the restriction mentioned above is invariant under the CS transformations; thus, the IWP solutions form a CS invariant class of solutions.

In Sec. 6 we formulate the most general technique for generation of new solutions based on the use of charging symmetries. We show, how starting from the pure Kaluza-Klein theory one can generate all the massless fields of the bosonic string theory and, next, the full heterotic string theory sector.

We conclude this work with a discussion about the charging symmetry subgroup and charging symmetry invariant in the non-perturbative regime of the heterotic string theory.

\section{Matrix Ernst Potentials}

Matrix Ernst potentials contain all information about the dynamical variables for the heterotic string theory reduced to three dimensions. These variables consist of ([4]-[5])

a) scalar fields

$G=\left(G_{p q}=G_{p+3, q+3}^{(D)}\right), \quad B=\left(B_{p q}=B_{p+3, q+3}^{(D)}\right), \quad A=\left(A_{p}^{I}=A_{p+3}^{(D) I}\right), \quad \phi=\phi^{(D)}-\frac{1}{2} \ln |\operatorname{det} G|$,

where the subscripts $p, q=1,2, \ldots, d$. 
b)tensor fields

$$
g_{\mu \nu}=e^{-2 \phi}\left(G_{\mu \nu}^{(D)}-G_{p+3, \mu}^{(D)} G_{q+3, \nu}^{(D)} G^{p q}\right), \quad B_{\mu \nu}=B_{\mu \nu}^{(D)}-4 B_{p q} A_{\mu}^{p} A_{\nu}^{q}-2\left(A_{\mu}^{p} A_{\nu}^{p+d}-A_{\nu}^{p} A_{\mu}^{p+d}\right),
$$

(we put $B_{\mu \nu}=0$ to remove the effective three-dimensional cosmological constant from our consideration $[10 \|)$.

c) vector fields $A_{\mu}^{(a)}=\left(\left(A_{1}\right)_{\mu}^{p},\left(A_{2}\right)_{\mu}^{p+d},\left(A_{3}\right)_{\mu}^{2 d+I}\right)(a=1, \ldots, 2 d+n)$

$\left(A_{1}\right)_{\mu}^{p}=\frac{1}{2} G^{p q} G_{q+3, \mu}^{(D)}, \quad\left(A_{3}\right)_{\mu}^{I+2 d}=-\frac{1}{2} A_{\mu}^{(D) I}+A_{q}^{I} A_{\mu}^{q}, \quad\left(A_{2}\right)_{\mu}^{p+d}=\frac{1}{2} B_{p+3, \mu}^{(D)}-B_{p q} A_{\mu}^{q}+\frac{1}{2} A_{p}^{I} A_{\mu}^{I+2 d}$.

These variables form a complete set of the $N=8$ supergravity in the critical case [10].

In three dimensions all vector fields can be dualized on-shell:

$$
\begin{aligned}
\nabla \times \overrightarrow{A_{1}} & =\frac{1}{2} e^{2 \phi} G^{-1}\left(\nabla u+\left(B+\frac{1}{2} A A^{T}\right) \nabla v+A \nabla s\right), \\
\nabla \times \overrightarrow{A_{3}} & =\frac{1}{2} e^{2 \phi}\left(\nabla s+A^{T} \nabla v\right)+A^{T} \nabla \times \overrightarrow{A_{1}}, \\
\nabla \times \overrightarrow{A_{2}} & =\frac{1}{2} e^{2 \phi} G \nabla v-\left(B+\frac{1}{2} A A^{T}\right) \nabla \times \overrightarrow{A_{1}}+A \nabla \times \overrightarrow{A_{3}} .
\end{aligned}
$$

The resulting three-dimensional theory describes the scalars $G, B, A$ and $\phi$ and pseudoscalars $u, v$ and $s$ coupled to the metric $g_{\mu \nu}$.

We define the matrix Ernst potentials as follows:

$$
\mathcal{X}=\left(\begin{array}{cc}
-e^{-2 \phi}+v^{T} X v+v^{T} A s+\frac{1}{2} s^{T} s & v^{T} X-u^{T} \\
X v+u+A s & X
\end{array}\right), \quad \text { and } \quad \mathcal{A}=\left(\begin{array}{c}
s^{T}+v^{T} A \\
A
\end{array}\right),
$$

where $X=G+B+\frac{1}{2} A A^{T}$. They are of the dimensions $(d+1) \times(d+1)$ and $(d+1) \times n$ correspondingly. In terms of MEP the effective three-dimensional theory takes the form:

$$
\begin{aligned}
S^{(3)} & =\int d^{3} x|g|^{\frac{1}{2}}\left\{-R+\operatorname{Tr}\left[\frac{1}{4}\left(\nabla \mathcal{X}-\nabla \mathcal{A} \mathcal{A}^{T}\right) \mathcal{G}^{-1}\left(\nabla \mathcal{X}^{T}-\mathcal{A} \nabla \mathcal{A}^{T}\right) \mathcal{G}^{-1}+\frac{1}{2} \nabla \mathcal{A}^{T} \mathcal{G}^{-1} \nabla \mathcal{A}\right]\right\} \\
& =\int d^{3} x|g|^{\frac{1}{2}}\left\{-R+\mathcal{L}_{H S}\right\},
\end{aligned}
$$

where

$$
\mathcal{G}=\frac{1}{2}\left(\mathcal{X}+\mathcal{X}^{T}-\mathcal{A} \mathcal{A}^{T}\right)
$$


It is well-known that the four-dimensional Einstein-Maxwell theory, being reduced to three dimensions, allows a similar formulation using two complex Ernst potentials $E$ and $F$ [9]. Let us rewrite them in the less conventional form

$$
\mathcal{X}_{E M}=\operatorname{Re} E+\sigma_{2} \operatorname{Im} E, \quad \mathcal{A}_{E M}=\operatorname{Re} F+\sigma_{2} \operatorname{Im} F, \quad \text { where } \quad \sigma_{2}=\left(\begin{array}{cc}
0 & -1 \\
1 & 0
\end{array}\right) .
$$

We can treat these matrices as the matrix Ernst potentials (2.4) of the $D=4$ theory (1.1) with $\phi^{(4)}=B_{M N}^{(4)}=0$. Then we conclude that $n=2$ and

$$
s^{1}=A^{2}=\operatorname{Re} F, \quad-s^{2}=A^{1}=\operatorname{Im} F .
$$

Note, that $s^{I}(I=1,2)$ describe the magnetic potentials, whereas $A^{I}$ are the electric ones. Thus, two Maxwell fields arising in the framework of the representation (2.5)-(2.8) occur to be mutually conjugated (i.e. $F_{M N}^{(4) 2}=\tilde{F}_{M N}^{(4) 1}$ in four dimensions). Next, for the single extra metric component one has:

$$
G=\frac{1}{2}(E+\bar{E}-F \bar{F}) \equiv f,
$$

whereas the rotational potential $u$ becomes equal to $\operatorname{Im} E$. Taking into account that $\mathcal{G}=G$, and substitute Eqs. (2.8), (2.10) into Eq. (2.6), we obtain

$$
\mathcal{L}_{E M}=\frac{1}{2 f^{2}}|\nabla E-\bar{F} \nabla F|^{2}+f^{-1}|\nabla F|^{2} .
$$

This is the matter Lagrangian of the three-dimensional EM theory [9]. Thus, our MEP formulation (2.5)-(2.7) of the HS theory includes the EM theory as a special case.

One can see that formally the transition from the HS theory (2.6) to the EM theory (2.11) is defined by the substitution

$$
\mathcal{X} \rightarrow E, \quad \mathcal{A} \rightarrow F
$$

together with the replacement

$$
\text { matrix transposition } \rightarrow \text { complex conjugation. }
$$

The inverse map (EM $\rightarrow$ HS) allow us to generalize the results for the EM theory, obtained using its complex representation, to the ones for the HS theory in the MEP formulation. This becomes possible if the matrix-valued generalized relation can be written in a form which is free of the matrix multipliers ordering problem. 


\section{Charging Symmetries}

The complete symmetry group (U-duality [6]) of the effective three-dimensional heterotic string theory (2.6) is $S O(d+1, d+1+n)$ [5]. Its action on the matrix Ernst potentials had been established in the our previous work [8]. There was shown that one discrete transformation, which is closely related to the so-called strong-weak coupling duality (we will call this transformation 'SWCD' [11]) plays a crucial role in the 'web' of HS symmetries (or dualities). Actually, the SWCD transformation

$$
\mathcal{X} \rightarrow \mathcal{X}^{-1} \quad \mathcal{A} \rightarrow-\mathcal{X}^{-1} \mathcal{A}
$$

maps the $\mathcal{X}$-shift symmetry

$$
\mathcal{X} \rightarrow \mathcal{X}+\lambda_{\mathcal{X}}, \quad \mathcal{A} \rightarrow \mathcal{A} \quad \text { where } \quad \lambda_{\mathcal{X}}^{T}=-\lambda_{\mathcal{X}}
$$

into the Ehlers transformation

$$
\mathcal{X} \rightarrow\left(1+\mathcal{X} \lambda_{\mathcal{E}}\right)^{-1} \mathcal{X}, \quad \mathcal{A} \rightarrow\left(1+\mathcal{X} \lambda_{\mathcal{E}}\right)^{-1} \mathcal{A}, \quad \text { where } \quad \lambda_{\mathcal{E}}^{T}=-\lambda_{\mathcal{E}} ;
$$

whereas the $\mathcal{A}$-shift symmetry

$$
\mathcal{A} \rightarrow \mathcal{A}+\lambda_{\mathcal{A}}, \quad \mathcal{X} \rightarrow \mathcal{X}+\mathcal{A} \lambda_{\mathcal{A}}^{T}+\frac{1}{2} \lambda_{\mathcal{A}} \lambda_{\mathcal{A}}^{T}
$$

maps into the Harrison transformation

$$
\mathcal{A} \rightarrow\left(1-\mathcal{A} \lambda_{\mathcal{H}}^{T}+\frac{1}{2} \mathcal{X} \lambda_{\mathcal{H}} \lambda_{\mathcal{H}}^{T}\right)^{-1}\left(\mathcal{A}-\mathcal{X} \lambda_{\mathcal{H}}\right), \quad \mathcal{X} \rightarrow\left(1-\mathcal{A} \lambda_{\mathcal{H}}^{T}+\frac{1}{2} \mathcal{X} \lambda_{\mathcal{H}} \lambda_{\mathcal{H}}^{T}\right)^{-1} \mathcal{X}
$$

The remaining symmetries, i.e. the electric-magnetic rotation

$$
\mathcal{A} \rightarrow \mathcal{A T}, \quad \mathcal{X} \rightarrow \mathcal{X}, \quad \text { where } \quad \mathcal{T} \mathcal{T}^{T}=1
$$

and the scaling transformation

$$
\mathcal{X} \rightarrow \mathcal{S}^{T} \mathcal{X} \mathcal{S}, \quad \mathcal{A} \rightarrow \mathcal{S}^{T} \mathcal{A}
$$

are the SWCD invariants (with $\mathcal{T} \rightarrow \mathcal{T}$ and $\mathcal{S} \rightarrow\left(\mathcal{S}^{T}\right)^{-1}$ ). Thus, U-duality of the threedimensional effective heterotic string theory consists of two doublets and two singlets of the strong-weak coupling duality transformation. 
Now let us consider the arbitrary constant potentials $\mathcal{X}=\mathcal{X}_{\infty}$ and $\mathcal{A}=\mathcal{A}_{\infty}$, which can have interpretation of the asymptotics near the spatial infinity. Applying the $\mathcal{A}$-shift symmetry with $\lambda_{\mathcal{A}}=-\mathcal{A}_{\infty}$ we annihilate the $\mathcal{A}$-potential. Next, using the $\mathcal{X}$-shift with $\lambda_{\mathcal{X}}=\left(\mathcal{X}_{\infty}^{T}-\mathcal{X}_{\infty}\right) / 2$ we remove the antisymmetric part of $\mathcal{X}$. Finally, the scaling with

$$
\mathcal{S}=\left(\begin{array}{cc}
e^{-\phi_{\infty}} & 0 \\
-e^{-\phi_{\infty}} v_{\infty}^{T} & \gamma_{\infty}^{-1}
\end{array}\right),
$$

where $G_{\infty}=\gamma_{\infty}^{T} \sigma \gamma_{\infty}$, leads $\mathcal{X}$ to the its trivial form

$$
\Sigma=\left(\begin{array}{cc}
-1 & 0 \\
0 & \sigma
\end{array}\right) .
$$

Here $\sigma=\operatorname{diag}(-1,1, \ldots, 1)$ is the tangent metric for $G_{\infty}$, whereas $\gamma_{\infty}$ is the tetrad matrix. Thus, U-duality contains gauge transformations which can be used for the removing of the all field asymptotics. Conversely, one can apply these 'dressing' transformations to obtain arbitrary asymptotics for the originally asymptoticaly-free field configuration. In the rest part of the paper we fix the gauge and put $\mathcal{X}_{\infty}=\Sigma$ and $\mathcal{A}_{\infty}=0$.

The transformations preserving fixed asymptotics form a subgroup, which we call 'charging' by the reasons given below. The electric-magnetic rotation belongs to this subgroup; another representative is related to the scaling transformation. Actually, the trivial $\mathcal{A}^{-}$ asymptotic preserves for the arbitrary value of $\mathcal{S}$; however only the scalings constrained by

$$
\mathcal{S}^{T} \Sigma \mathcal{S}=\Sigma
$$

do not change the chosen $\mathcal{X}$-asymptotic. Thus, the group of charging symmetries contains the $S O(2, d-1)$ subgroup of the scaling symmetry.

One can see that the Ehlers transformation with the arbitrary non-trivial parameter $\lambda_{\mathcal{E}}$ moves the asymptotical value $\mathcal{X}_{\infty}=\Sigma$. However, some combination of the Ehlers transformation with the special $\mathcal{X}$-shift and scaling duality belongs to the charging symmetry subgroup. Actually, let us suppose that the Ehlers transformation with the arbitrary antisymmetric parameter $\lambda_{\mathcal{X}}$ is applied to the matrices $\mathcal{X}_{\infty}=\Sigma$ and $\mathcal{A}_{\infty}=0$. Then the value of $\mathcal{A}_{\infty}$ remains trivial, whereas $\mathcal{X}_{\infty}$ becomes changed. Then, to remove the antisymmetric part of new $\mathcal{X}_{\infty}$, we perform the $\mathcal{X}$-shift transformation with $\lambda_{\mathcal{X}}=\left(1+\Sigma \lambda_{\mathcal{E}}\right)^{-1} \Sigma \lambda_{\mathcal{E}} \Sigma\left(1-\lambda_{\mathcal{E}} \Sigma\right)^{-1}$. Finally, we transform the resulting $\mathcal{X}_{\infty}$-asymptotic to $\Sigma$ using the scaling (3.7) with $\mathcal{S}=1-\lambda_{\mathcal{E}} \Sigma$. The resulting transformation has the form

$$
\begin{aligned}
\mathcal{X} & \rightarrow\left(1+\Sigma \lambda_{\mathcal{E}}\right)\left(1+\mathcal{X} \lambda_{\mathcal{E}}\right)^{-1} \mathcal{X}\left(1-\lambda_{\mathcal{E}} \Sigma\right)+\Sigma \lambda_{\mathcal{E}} \Sigma \\
\mathcal{A} & \rightarrow\left(1+\Sigma \lambda_{\mathcal{E}}\right)\left(1+\mathcal{X} \lambda_{\mathcal{E}}\right)^{-1} \mathcal{A} .
\end{aligned}
$$


We call this symmetry normalized Ehlers transformation (NET).

The similar 'normalization' procedure can be performed for the Harrison transformation with the arbitrary matrix parameter $\lambda_{\mathcal{H}}$. First, we remove the $\mathcal{A}$-asymptotic generated by the Harrison transformation using the $\mathcal{A}$-shift symmetry with $\lambda_{\mathcal{A}}=\left(1+\Sigma \frac{1}{2} \lambda_{\mathcal{H}} \lambda_{h}^{T}\right)^{-1} \Sigma \lambda_{\mathcal{H}}$. Second, we transform the 'broken' $\mathcal{X}$-asymptotic to $\Sigma$ using the scaling with $\mathcal{S}=1+$ $\frac{1}{2} \Sigma \lambda_{\mathcal{H}} \lambda_{\mathcal{H}}^{T}$. The resulting normalized Harrison transformation (NHT) is defined by the formulae

$$
\begin{aligned}
& \mathcal{A} \rightarrow\left(1+\frac{1}{2} \Sigma \lambda_{\mathcal{H}} \lambda_{\mathcal{H}}^{T}\right)\left(1-\mathcal{A} \lambda_{\mathcal{H}}^{T}+\frac{1}{2} \mathcal{X} \lambda_{\mathcal{H}} \lambda_{\mathcal{H}}^{T}\right)^{-1}\left(A-\mathcal{X} \lambda_{\mathcal{H}}\right)+\Sigma \lambda_{\mathcal{H}} \\
& \mathcal{X} \rightarrow\left(1+\frac{1}{2} \Sigma \lambda_{\mathcal{H}} \lambda_{\mathcal{H}}^{T}\right)\left(1-\mathcal{A} \lambda_{\mathcal{H}}^{T}+\frac{1}{2} \mathcal{X} \lambda_{\mathcal{H}} \lambda_{\mathcal{H}}^{T}\right)^{-1}\left[\mathcal{X}+\left(\mathcal{A}-\frac{1}{2} \mathcal{X} \lambda_{\mathcal{H}}\right) \lambda_{\mathcal{H}}^{T} \Sigma\right]+\frac{1}{2} \Sigma \lambda_{\mathcal{H}} \lambda_{\mathcal{H}}^{T} \Sigma
\end{aligned}
$$

The number of the dressing symmetries is equal to the number of the dynamical variables of HS theory (2.6) , i.e. to $(d+1)(d+1+n)$. The electric-magnetic rotations (3.6) form the $\mathrm{SO}(\mathrm{n})$ subgroup defined by $n(n-1) / 2$ parameters, whereas the $S O(2, d-1)$ scaling subgroup (3.7), as well as NET (3.11), gives $(d+1) d / 2$ parameters. Finally, the NHT is related to the parameter matrix with $(d+1) n$ elements. Thus, all the established transformations from the CS subgroup, being independent, are constructed from $(d+1)(d+n)+n(n-1) / 2$ parameters. Then the common number of independent dressing and charging transformations becomes equal to $[2(d+1)+n][2(d+1)+n-1] / 2$, i.e. to the number of parameters of the whole U-duality group $S O(d+1, d+1+n)$. From this it follows that we have found all the gauge (dressing) transformations as well as all the non-gauge (charging) symmetries. Thus, the CS subgroup consists of the electric-magnetic rotation (3.6), the scaling (3.7) restricted by Eq. (3.10), and the normalized Ehlers (3.11) and Harrison (3.12) transformations.

In the EM theory case the $\mathcal{X}$-shift, Ehlers, electric-magnetic and scaling transformations become one-parametric symmetries; they are defined by the linear combinations of the twodimensional unit matrix and $\sigma_{2}$ (see Eqs. (3.2), (3.3), (3.6), (3.7) and (3.10)). To preserve the same structure of the matrix Ernst potentials (2.8) under the action of the remaining two symmetries, we must suppose that the $\mathcal{A}$-shift and Harrison transformations are defined by two-parametric linear combinations of the unit and $\sigma_{2}$ matrices. Finally, the general symmetry group ('U-duality') of the EM theory in three dimensions occurs to be eightparametric. This really takes place because this group is isomorphic to $S U(1,2)$ [12].

Using the substitution (2.12) it is easy to rewrite all the symmetries in the conventional form of the complex Ernst potentials (the matrix $\sigma_{2}$ must be changed to the imaginary unit i). Below we will need only in the CS transformations; they can be obtained from Eqs. (3.6), (3.7), (3.10), (3.11) and (3.12) using the additional replacement

$$
\Sigma \rightarrow-1
$$


(we consider the time compactification for definiteness )

$$
\begin{gathered}
E \rightarrow E, \quad F \rightarrow e^{i \alpha} F ; \quad(\mathrm{EMT}) \\
E \rightarrow \frac{E+i \epsilon}{1+i \epsilon E}, \quad F \rightarrow \frac{1-i \epsilon}{1+i \epsilon E} F ; \quad(\mathrm{NET}) \\
E \rightarrow \frac{E+\frac{1}{2}\left|\lambda_{\mathcal{H}}\right|^{2}-\bar{\lambda}_{\mathcal{H}} F}{1-\bar{\lambda}_{\mathcal{H}} F+\frac{1}{2}\left|\lambda_{\mathcal{H}}\right|^{2} E}, \quad F \rightarrow \frac{\left(1+\frac{1}{2}\left|\lambda_{\mathcal{H}}\right|^{2}\right) F-\lambda_{\mathcal{H}}(E+1)}{1-\bar{\lambda}_{\mathcal{H}} F+\frac{1}{2}\left|\lambda_{\mathcal{H}}\right|^{2} E}, \quad \text { (NHT) }
\end{gathered}
$$

In Eq. (3.16) the parameter $\lambda_{\mathcal{H}}$ is complex; the other two parameters $\alpha$ and $\epsilon$ are real. From Eqs. (3.6), (3.7) and (3.10) it follows that NST and EMT coincide in the case of a single Maxwell field. We call the corresponding transformation 'electric-magnetic transformation' because this name remains natural for the case of $n>1$.

\section{Linearizing Potentials}

One can see that the electric-magnetic rotation and scaling act as linear transformations on the matrix Ernst potentials $\mathcal{X}$ and $\mathcal{A}$, whereas the normalized Ehlers and Harrison transformations are some fractional-linear maps. In this section we introduce new matrix potentials $\mathcal{Z}_{1}$ and $\mathcal{Z}_{2}$ which linearly transform under the action of the all CS transformations, i.e. they form a possible set of CS linearizing potentials.

In [13] we have found the linearizing potentials for the special case of $d=n=1$ (the so-called Einstein-Maxwell dilaton-axion (EMDA) theory). This theory allows a Kähler representation using three complex Ernst potentials [14]. First, we constructed a linear realization of the CS subgroup on the set of three over complex variables. Second, we computed all the commutation relations between the CS generators in the both representations and, finally, we identify the generators accordingly to their commutation relations. This identification gave us differential equations of the first order which define the unknown functional relations between the Ernst and linearizing potentials. The result of [13 can easily be extended to the case of $d=1$ and arbitrary $n$. However, the following extension to the case of $d>1$ seems impossible because the corresponding Kähler formulation for the theory is absent.

To construct the linearizing potentials for the arbitrary $d$ and $n$ (including the critical case of $d=7, n=16$ ), first we will find them for the EM theory in its complex representation. 
After that, using the map (2.12), (3.13) in its inverse form, we will obtain LP in the general case.

In the Appendix A one can find the details of the LP derivation for the Einstein-Maxwell theory using the strategy formulated above for the EMDA theory. The result is:

$$
\mathcal{Z}_{1}=2(E-1)^{-1}+1, \quad \mathcal{Z}_{2}=\sqrt{2}(E-1)^{-1} F \quad(\text { EM } \quad \text { theory })
$$

One can see that these equations admit the straightforward HS generalization, because the problem of the matrix multiplier ordering does not arise. Then

$$
\left.\mathcal{Z}_{1}=2(\mathcal{X}+\Sigma)^{-1}-\Sigma, \quad \mathcal{Z}_{2}=\sqrt{2}(\mathcal{X}+\Sigma)^{-1} \mathcal{A} \quad \text { (HS theory }\right) .
$$

One can prove that the inverse relations can be obtained using the replacements $\mathcal{Z}_{1} \leftrightarrow \mathcal{X}$ and $\mathcal{Z}_{2} \leftrightarrow \mathcal{A}$. This means that the inverse functions of the functions (4.2) coincide with them. Actually, this remarkable property fixes the multiplier ' $\sqrt{2}$ '.

We have supposed that the fractional-linear functions of Eq. (4.2) define the linearizing potentials for the HS theory. To verify this, it is necessary to rewrite all the CS transformations using the $\left(\mathcal{Z}_{1}, \mathcal{Z}_{2}\right)$-representation. The result is:

$$
\begin{gathered}
\mathcal{Z}_{1} \rightarrow \mathcal{Z}_{1}, \quad \mathcal{Z}_{2} \rightarrow \mathcal{Z}_{2} \mathcal{T} ; \quad(\text { EMT }) \\
\mathcal{Z}_{1} \rightarrow \mathcal{S}^{-1} \mathcal{Z}_{1}\left(\mathcal{S}^{T}\right)^{-1}, \quad \mathcal{Z}_{2} \rightarrow \mathcal{S}^{-1} \mathcal{Z}_{2} ; \quad \text { (scaling) } \\
\mathcal{Z}_{1} \rightarrow \mathcal{Z}_{1}\left(1-\Sigma \lambda_{\mathcal{E}}\right)\left(1+\Sigma \lambda_{\mathcal{E}}\right)^{-1}, \quad \mathcal{Z}_{2} \rightarrow \mathcal{Z}_{2} ; \quad \text { (NET) } \\
\mathcal{Z}_{1} \rightarrow \mathcal{Z}_{1}\left(1-\frac{1}{2} \Sigma \lambda_{\mathcal{H}} \lambda_{\mathcal{H}}^{T}\right)\left(1+\frac{1}{2} \Sigma \lambda_{\mathcal{H}} \lambda_{\mathcal{H}}^{T}\right)^{-1}-\sqrt{2} \mathcal{Z}_{2} \lambda_{\mathcal{H}}^{T}\left(1+\frac{1}{2} \Sigma \lambda_{\mathcal{H}} \lambda_{\mathcal{H}}^{T}\right)^{-1}, \\
\mathcal{Z}_{2} \rightarrow \mathcal{Z}_{2}\left[1-\lambda_{\mathcal{H}}^{T}\left(\Sigma+\frac{1}{2} \lambda_{\mathcal{H}} \lambda_{\mathcal{H}}^{T}\right)^{-1} \lambda_{\mathcal{H}}\right]+\sqrt{2} \mathcal{Z}_{1}\left(\Sigma+\frac{1}{2} \lambda_{\mathcal{H}} \lambda_{\mathcal{H}}^{T}\right)^{-1} \lambda_{\mathcal{H}},
\end{gathered}
$$

where 'EMT' denotes the electric-magnetic transformation. From these formulae we see that the linearization really takes place. Thus, $\mathcal{Z}_{1}$ and $\mathcal{Z}_{2}$ actually form a set of linearizing potentials of the charging symmetry subgroup of the three-dimensional heterotic string theory (2.6).

The linearizing potentials allow us to clarify the CS subgroup structure. Actually, the defining NET matrix

$$
\mathcal{E}=\left(1-\Sigma \lambda_{\mathcal{E}}\right)\left(1+\Sigma \lambda_{\mathcal{E}}\right)^{-1}
$$


satisfies the $S O(2, d-1)$ group relation $\mathcal{E}^{T} \Sigma \mathcal{E}=\Sigma$. Conversely, it is easy to see that any $S O(2, d-1)$-matrix can be written in the form of Eq. (4.7). Thus, the normalized Ehlers transformation forms the $S O(2, d-1)$ CS subgroup. Next, NET can be explored for some 'normalization' of the scaling symmetry subgroup. Actually, these both transformations are represented by the matrices of the $S O(2, d-1)$ group. We define the normalized scaling transformation (NST) as result of the action of the scaling with $\mathcal{S} \in S O(2, d-1)$ and NET with $\mathcal{E}=\mathcal{S}^{T}$ :

$$
\mathcal{Z}_{1} \rightarrow \mathcal{S}^{-1} \mathcal{Z}_{1}, \quad \mathcal{Z}_{2} \rightarrow \mathcal{S}^{-1} \mathcal{Z}_{2} . \quad(\mathrm{NST})
$$

The significance of the scaling subgroup normalization is related with the fact that NST commutes with all other CS transformations, because NST acts as the left multiplier on $\mathcal{Z}_{1}$ and $\mathcal{Z}_{2}$ whereas EMT, NET and NHT are the right multipliers (see Eqs. (4.3), (4.5), (4.6) and $(4.8))$.

To analyse the group structure of the right multiplier sector (the single left multiplier forms the $S O(2, d-1)$ subgroup) we will need in one general CS invariant. This invariant can be 'extracted' from the Lagrangian $\mathcal{L}_{H S}$ (see Eq. (2.6)). Actually, let us consider the asymptotically trivial fields with the non-zero Coulomb terms:

$$
\mathcal{Z}_{1}=\frac{\mathcal{Q}_{1}}{r}+o\left(\frac{1}{r}\right), \quad \mathcal{Z}_{2}=\frac{\mathcal{Q}_{2}}{r}+o\left(\frac{1}{r}\right)
$$

where $\mathcal{Q}_{1}$ and $\mathcal{Q}_{2}$ are the charge matrices and $r$ tends to the spatial infinity. Then, using the inverse to Eqs. (4.2) relations we obtain that

$$
\mathcal{L}_{H S}=\frac{1}{r^{4}} \operatorname{Tr}\left\{\mathcal{Q}_{1}^{T} \Sigma \mathcal{Q}_{1} \Sigma+\mathcal{Q}_{2}^{T} \Sigma \mathcal{Q}_{2}\right\}+o\left(\frac{1}{r^{4}}\right) .
$$

The quadratic charge combination $\mathcal{I}=\operatorname{Tr}\left\{\mathcal{Q}_{1}^{T} \Sigma \mathcal{Q}_{1} \Sigma+\mathcal{Q}_{2}^{T} \Sigma \mathcal{Q}_{2}\right\}$ is the CS invariant. It is so because $\mathcal{L}_{H S}$ is the CS invariant and all its terms related to the $1 / r$ power expansion are also CS invariants. Now it is useful to introduce the $(d+1) \times(d+1+n)$ charge matrix $\mathcal{Q}=\left(\mathcal{Q}_{1}, \mathcal{Q}_{2}\right)$ and to rewrite $\mathcal{I}$ in the form

$$
\mathcal{I}(\mathcal{Q})=\operatorname{Tr}\left\{\mathcal{Q}^{T} \Sigma \mathcal{Q} \Xi\right\}, \quad \text { where } \quad \Xi=\left(\begin{array}{cc}
\Sigma & 0 \\
0 & 1
\end{array}\right) .
$$

Let us now introduce the $(d+1) \times(d+1+n)$ linearizing potential $\mathcal{Z}=\left(\mathcal{Z}_{1}, \mathcal{Z}_{2}\right)$. Then, from Eq. (4.9) it follows that the charge and linearizing potential matrices have the same transformation properties. Thus, the function

$$
\mathcal{I}(\mathcal{Z})=\operatorname{Tr}\left\{\mathcal{Z}^{T} \Sigma \mathcal{Z} \Xi\right\}
$$


must be a charging symmetry invariant.

All the charging symmetry transformations can be rewritten in the form

$$
\mathcal{Z} \rightarrow \mathcal{G}_{i}^{\text {left }} \mathcal{Z G}_{i}^{\text {right }},
$$

where $i=\mathrm{NST}$, EMT, NET or NHT and an explicit form of the matrices $\mathcal{G}_{i}^{\text {left }}$ and $\mathcal{G}_{i}^{\text {right }}$ can be obtained from Eqs. (4.3), (4.5), (4.6) and (4.8). The only non-unit matrices are:

$$
\begin{aligned}
& \mathcal{G}_{\text {NST }}^{\text {left }}=\left(\begin{array}{cc}
\mathcal{S}^{-1} & 0 \\
0 & 1
\end{array}\right), \quad \mathcal{G}_{\mathrm{EMT}}^{\text {right }}=\left(\begin{array}{cc}
1 & 0 \\
0 & \mathcal{T}
\end{array}\right), \quad \mathcal{G}_{\mathrm{NET}}^{\text {right }}=\left(\begin{array}{cc}
\mathcal{E} & 0 \\
0 & 1
\end{array}\right), \\
& \mathcal{G}_{\mathrm{NHT}}^{\text {right }}=\left(\begin{array}{cc}
\left(1-\frac{1}{2} \Sigma \lambda_{\mathcal{H}} \lambda_{\mathcal{H}}^{T}\right)\left(1+\frac{1}{2} \Sigma \lambda_{\mathcal{H}} \lambda_{\mathcal{H}}^{T}\right)^{-1} & \sqrt{2}\left(\Sigma+\frac{1}{2} \lambda_{\mathcal{H}} \lambda_{\mathcal{H}}^{T}\right)^{-1} \lambda_{\mathcal{H}} \\
-\sqrt{2} \lambda_{\mathcal{H}}^{T}\left(1+\frac{1}{2} \Sigma \lambda_{\mathcal{H}} \lambda_{\mathcal{H}}^{T}\right)^{-1} & 1-\lambda_{\mathcal{H}}^{T}\left(\Sigma+\frac{1}{2} \lambda_{\mathcal{H}} \lambda_{\mathcal{H}}^{T}\right)^{-1} \lambda_{\mathcal{H}}
\end{array}\right) .
\end{aligned}
$$

To preserve $\mathcal{I}(\mathcal{Z})$ all the 'left' transformations must satisfy the relation

$$
\left(\mathcal{G}_{i}^{\text {left }}\right)^{T} \Sigma \mathcal{G}_{i}^{\text {left }}=\Sigma
$$

whereas all the 'right' transformations must be constrained accordingly to

$$
\mathcal{G}_{i}^{\text {right }} \Xi\left(\mathcal{G}_{i}^{\text {right }}\right)^{T}=\Xi .
$$

The first relation really takes place (see Eq. (3.10)); this means that $\mathcal{G}_{i}^{\text {left }} \in S O(2, d-1)$. The direct substitution of the 'right' matrices from Eqs. (4.14) to Eq. (4.16) gives the identity. Taking into account the explicit form of $\Xi$ we conclude that all the transformations $\mathcal{G}_{i}^{\text {right }} \in S O(2, d-1+n)$.

Now let us note that the common number of independent parameters of EMT, NET and NHT is $(d+1+n)(d+n) / 2$, i.e. the same one as for the $S O(2, d-1+n)$ group. Moreover, if we consider the infinitesimal transformations $\mathcal{G}_{i}^{\text {right }}=1+\Gamma_{i}^{\text {right }}$ and compute $\Gamma^{\text {right }}=\sum_{i} \Gamma_{i}$, we obtain

$$
\Gamma^{r i g h t}=\left(\begin{array}{cc}
-2 \Sigma \lambda_{\mathcal{E}} & \sqrt{2} \Sigma \lambda_{\mathcal{H}} \\
-\sqrt{2} \lambda_{\mathcal{H}}^{T} & \tau
\end{array}\right)
$$

where $\tau=\mathcal{T}-1$. This matrix is the general solution of the equation $\left(\Gamma^{\text {right }}\right)^{T}=-\Xi \Gamma^{\text {right }} \Xi$, which defines the $s o(2, d-1+n)$ algebra (its structure is discussed in the Appendix B). From this we conclude that the general 'right' transformation matrix is the general matrix 
of the group $S O(2, d-1+n)$. It can be constructed as the product of the all $\mathcal{G}_{i}^{\text {right }}$ matrices multiplied in an arbitrary order.

Thus, we have established the following simplest form of the charging symmetry transformations for the effective three-dimensional heterotic string theory at low energies:

$$
\mathcal{Z} \rightarrow \mathcal{G}^{\text {left }} \mathcal{Z} \mathcal{G}^{\text {right }}, \quad \text { where } \quad \mathcal{G}^{\text {left }} \in S O(2, d-1) \quad \text { and } \quad \mathcal{G}^{\text {right }} \in S O(2, d-1+n) .
$$

The charging symmetry subgroup occurs to be $\mathcal{G}^{\text {left }} \times \mathcal{G}^{\text {right }}$, i.e. it is isomorphic to $S O(2, d-$ 1) $\times S O(2, d-1+n)$. It is important to note that the transformation of the charge matrix $\mathcal{Q}$ can be obtained from Eq. (4.18) using the replacement $\mathcal{Z} \rightarrow \mathcal{Q}$.

The strong-weak coupling duality transformation in terms of the linearizing potential $\mathcal{Z}$ takes the form:

$$
\mathcal{Z} \rightarrow-\Sigma \mathcal{Z} \Xi
$$

From Eqs. (4.18) and (4.19) it follows that the SWCD acts on the subgroups $\mathcal{G}^{\text {left }}$ and $\mathcal{G}^{\text {right }}$ in the following way:

$$
\begin{aligned}
& \mathcal{G}^{\text {left }} \rightarrow \Sigma \mathcal{G}^{\text {left }} \Sigma \\
& \mathcal{G}^{\text {right }} \rightarrow \Xi \mathcal{G}^{\text {right }} \Xi
\end{aligned}
$$

One can see that these maps preserve the group relations (4.15) and (4.16). Thus, both $\mathcal{G}^{\text {left }}$ and $\mathcal{G}^{\text {right }}$ are the SWCD-invariant. This means, that the whole CS subgroup is also SWCD invariant. Taking into account that all the dressing symmetries do not possess this property we obtain the following alternative definition of the CS subgroup: the charging symmetry subgroup is the maximal subgroup of the $U$-duality which is invariant under the action of the SWCD transformation.

\section{Invariant Fields}

Charging symmetries act as linear homogeneous transformations on the linearizing potentials. Let us consider the simplest linear homogeneous field configuration

$$
\mathcal{Z}_{2}=\mathcal{Z}_{1} \mathcal{B}
$$

where $\mathcal{B}$ is the constant $(d+1) \times n$ matrix. It is easy to see that this configuration preserves its form under the action of charging symmetries. Thus, Eq. (5.1) describes the charging symmetry invariant class of fields. The transformation lows for the matrix $\mathcal{B}$ are:

$$
\mathcal{B} \rightarrow \mathcal{B} \text { for NST, } \quad \mathcal{B} \rightarrow \mathcal{B T} \quad \text { for EMT }, \quad \mathcal{B} \rightarrow \mathcal{E}^{-1} \mathcal{B} \quad \text { for NET; }
$$


whereas for NHT one has

$$
\begin{gathered}
\mathcal{B} \rightarrow\left(1+\frac{1}{2} \Sigma \lambda_{\mathcal{H}} \lambda_{\mathcal{H}}^{T}\right)\left(1-\frac{1}{2} \Sigma \lambda_{\mathcal{H}} \lambda_{\mathcal{H}}^{T}-\sqrt{2} \quad \mathcal{B} \lambda_{\mathcal{H}}^{T}\right)^{-1}\left\{\mathcal{B}\left[1-\lambda_{\mathcal{H}}^{T}\left(\Sigma+\frac{1}{2} \lambda_{\mathcal{H}} \lambda_{\mathcal{H}}^{T}\right)^{-1} \lambda_{\mathcal{H}}\right]\right. \\
\left.+\sqrt{2}\left(\Sigma+\frac{1}{2} \lambda_{\mathcal{H}} \lambda_{\mathcal{H}}^{T}\right)^{-1} \lambda_{\mathcal{H}}\right\}
\end{gathered}
$$

Eqs. (5.2) and (5.3) describe the realization of the CS subgroup on the matrix $\mathcal{B}$ (which is trivial for $\mathcal{G}^{\text {left }}$ and non-trivial for $\mathcal{G}^{\text {right }}$ ).

For the EM theory in its complex representation $\mathcal{B}$ becomes the complex parameter. EMT and NET occur to be equal; they are given by the factor $\mathcal{T}=\mathcal{E}^{-1}=\exp (i \delta)$. The corresponding generator is

$$
\Gamma_{N E T}=i \mathcal{B} \partial_{\mathcal{B}}
$$

Next, NHT consists of two real transformations; decomposing its complex parameter to the real and imaginary parts, $\lambda_{\mathcal{H}}=\frac{1}{2 \sqrt{2}}\left(\lambda_{\mathcal{H}_{1}}+i \lambda_{\mathcal{H}_{2}}\right)$, we obtain:

$$
\begin{aligned}
\Gamma_{N H T_{1}} & =\frac{1}{2}\left(\mathcal{B}^{2}-1\right) \partial_{\mathcal{B}}, \\
\Gamma_{N H T_{2}} & =-\frac{i}{2}\left(\mathcal{B}^{2}+1\right) \partial_{\mathcal{B}}
\end{aligned}
$$

(we write down only the holomorphic terms). Now it is easy to verify that the generators (5.4)-(5.5) satisfy the commutation relations of the $s u(1,1)$ algebra:

$$
\left[\Gamma_{N H T_{1}}, \Gamma_{N E T}\right]=\Gamma_{N H T_{2}}, \quad\left[\Gamma_{N E T}, \Gamma_{N H T_{2}}\right]=\Gamma_{N H T_{1}}, \quad\left[\Gamma_{N H T_{1}}, \Gamma_{N E T_{2}}\right]=-\Gamma_{N E T} .
$$

Next, in the general HS theory case the charging symmetry invariant, being computed for the fields $(5.1)$, is equal to $\mathcal{I}(\mathcal{Z})=\operatorname{Tr}\left[\mathcal{Z}_{1}^{T} \Sigma \mathcal{Z}_{1}\left(\mathcal{B B}^{T}+\Sigma\right)\right]$; it vanishes if

$$
\mathcal{B B}^{T}=-\Sigma \text {. }
$$

It is easy to see that this restriction is invariant itself under the action of NST, EMT and NET. After some algebraic manipulations one can prove that NHT (5.3) also preserves Eq. (5.7). Thus, the restriction (5.7) is invariant under the all charging symmetry transformations.

In 15 it was shown (in terms of $\mathcal{X}$ and $\mathcal{A}$ ) that the field configuration (5.1) satisfies the motion equations of the theory if it is restricted by Eq. (5.7). It describes the Israel-WilsonPerj'es (IWP) class of solutions for the HS theory reduced to three dimensions. As the 
consequence of our consideration we conclude that the IWP solutions form the CS invariant class. This means that the complete CS subgroup $S O(2, d-1) \times S O(2, d-1+n)$ is the group of invariance for the IWP solution. Moreover, if one interested in the arbitrary asymptotics, one must perform the dressing transformations to obtain the $U$-duality invariant class.

In the EM theory case the restriction (5.7) means that $\mathcal{B}=\exp (i \beta)$, where $\beta$ is a real parameter. Using the usual procedure one can compute the NET and NHT generators; the result is:

$$
\Gamma_{N E T}=\partial_{\beta}, \quad \Gamma_{N H T_{1}}=\sin \beta \partial_{\beta}, \quad \Gamma_{N H T_{2}}=-\cos \beta \partial_{\beta} .
$$

These generators also form the $s u(1,1)$ algebra, its arising (instead of the only expected $u(1)$ algebra) is related with the non-trivial action of NHT on the parameter $\beta$ and on the independent linearizing potential $\mathcal{Z}_{1}$. Thus, we have established that the IWP solutions form the CS invariant class in the EM and HS theories.

\section{Generation Technique}

In a fact, starting from the arbitrary solution and performing all the CS (or U-duality) transformations one obtains the solution class which is CS (or U-duality) invariant. Let us consider the generation of the HS asimptoticaly-free solutions, which can be 'dressed' in a usual way. This process becomes simplest and manifestly CS-invariant in terms of the linearizing potential $\mathcal{Z}$ (see Eq. (4.18)). The only question is related to the choice of class of seed solutions.

It is natural to start with the $(d+3)$-dimensional Kaluza-Klein $(\mathrm{KK})$ theory. Being reduced to three dimensions, this theory is described by the single matrix Ernst potential (see Eqs. (2.1)-(2.5))

$$
\mathcal{X}_{K K}=\left(\begin{array}{cc}
\operatorname{det} G & -u^{T} \\
u & G
\end{array}\right) .
$$

The $\mathcal{X}_{K K}$ block elements can be combined into the $S L(d+1, R) / S O(d+1)$ coset matrix 16

$$
\mathcal{M}_{K K}=\left(\begin{array}{cc}
G^{-1} & G^{-1} u \\
u^{T} G^{-1} & \operatorname{det} G+u^{T} G^{-1} u
\end{array}\right) .
$$

In its terms $\mathcal{L}_{K K}=-\frac{1}{4} \operatorname{Tr}\left(\nabla \mathcal{M}_{K K} \nabla \mathcal{M}_{K K}^{-1}\right)$, i.e. the KK theory obtains a chiral form. This form allowed to obtain wide classes of the three-dimensional solutions defined by a set 
of harmonic functions (see [17]), etc. Moreover, in two dimensions the inverse scattering transform technique leads to construction of the $2 \mathrm{~N}$-soliton solution (see 18 for the $d=1$ and $d=2$ cases).

One can generate the HS theory solutions from the KK ones step-by-step. Actually, one can apply NST and NET to the seed solution $\mathcal{Z}_{1_{K K}}$ (constructed from the known matrix $\left.\mathcal{X}_{K K}\right)$. The result is the potential $\mathcal{Z}_{1_{B}}$, which contains not only the metric, but also the dilaton and Kalb-Ramond fields. These are all the degrees of freedom of the bosonic string theory, i.e. the normalized scaling and Ehlers transformations map the Kaluza-Klein theory into the bosonic string one. Next, an applying of the NHT to the potential $\mathcal{Z}_{1_{B}}$ gives the whole sector of the HS theory $\mathcal{Z}_{1_{H S}}$ and $\mathcal{Z}_{2_{H S}}$. This means that the normalized Harrison transformation maps the bosonic string theory into the heterotic string one. Finally, EMT mixes the $\mathcal{Z}_{2_{H S}}$ components. Thus, we lead to the following generation technique:

$$
\text { Kaluza-Klein } \stackrel{\text { NET, NST }}{\Longrightarrow} \text { Bosonic String } \stackrel{\text { NHT, EMT }}{\Longrightarrow} \text { Heterotic String }
$$

If the seed KK solution has the Coulomb term in its $1 / r$-expansion, the above described process simultaneously generates the charges: NET and NST generate the dilaton and KalbRamond charges, whereas NHT and EMT generate the electric and magnetic ones. All these properties explain the name 'charging' for the symmetries preserving field asymptotics.

\section{Concluding Remarks}

Thus, we have extracted all the charging symmetries from the U-duality group of the effective three-dimensional theory of heterotic string. We have established the linearizing potentials which undergo linear homogeneous transformations when the charging symmetries act. We have constructed one general invariant of these symmetries, quadratic on the linearizing potentials.

It is shown that the charging symmetries preserve the linear homogeneous relation between the linearizing potentials. It is established that the corresponding anzats defines the class of Israel-Wilson-Perj'es solutions in the special case when the above mentioned invariant vanishes. Furthermore, the condition extracting IWP solution from the linear homogeneous anzats occurs to be CS-invariant itself. Thus, it is stressed the close relation between the charging symmetries, the linearizing potentials and the IWP solutions.

It is well known that the IWP solutions describe supersymmetric field configurations in any gravity theory which can be embedded into some supergravity. For example, this takes 
place for the arbitrary EM theory [19] and for the EM theory with the dilaton and axion fields [20]. In the forthcoming paper we hope to perform the corresponding analysis for the IWP solution in the critical HS theory.

In the non-perturbative case the charges of field configuration become quantized accordingly to the Dirac-Schwinger-Zwanziger rule [21]. The IWP solution, being supersymmetric, does not obtain quantum corrections to its charges, which become integer. The CS subgroup, as its group of invariance, become the integer-valued group $S O(2, d-1 ; Z) \times S O(2, d-1+$ $n ; Z)$.

The general charging symmetry invariant vanishes for the IWP solution in the perturbative regime. The first non-trivial term in the CSI asymptotic expansion near to the spatial infinity remains vanishing in the non-perturbative regime as the BPS-bound for the supersymmetric solution. It seems natural that CSI preserves its zero value in the whole three-dimensional space for the supersymmetric IWP solutions in the non-perturbative sector of HS theory.

\section{Acknowledgments}

We thank our colleagues for encouraging us. A.H. was partially supported by CONACYT.

\section{Appendix A: LP for EM}

In this Appendix we derive the linearizing potentials of the charging symmetry subgroup for the stationary Einstein-Maxwell theory.

The generators of the CS subgroup can be obtained from Eqs. (3.14)-(3.16); the result is:

$$
\begin{gathered}
\Gamma_{N H T_{1}}=F(E-1) \partial_{E}+\left(F^{2}-E-1\right) \partial_{F}, \quad \Gamma_{N H T_{2}}=-i\left[F(E-1) \partial_{E}+\left(F^{2}+E+1\right) \partial_{F}\right] . \\
\Gamma_{N E T}=i\left[\left(1-E^{2}\right) \partial_{E}-F(E+1) \partial_{F}\right], \quad \Gamma_{E M T}=i F \partial_{F},
\end{gathered}
$$

Here $\Gamma_{N H T_{1}}\left(\Gamma_{N H T_{2}}\right)$ corresponds to the real (imaginary) part of the Harrison's parameter $\lambda_{\mathcal{H}}$. It is useful to introduce the new set of generators

$$
X_{1}=-\frac{1}{2 \sqrt{2}} \Gamma_{N H T_{1}}, \quad X_{2}=\frac{1}{2 \sqrt{2}} \Gamma_{N H T_{2}},
$$




$$
X_{3}=\frac{1}{4}\left(2 \Gamma_{E M T}-\Gamma_{N E T}\right), \quad X_{4}=\frac{1}{4}\left(2 \Gamma_{E M T}+\Gamma_{N E T}\right)
$$

then

$$
\left[X_{1}, X_{2}\right]=-X_{3}, \quad\left[X_{2}, X_{3}\right]=X_{1}, \quad\left[X_{3}, X_{1}\right]=X_{2},
$$

and $\left[X_{1}, X_{4}\right]=\left[X_{2}, X_{4}\right]=\left[X_{3}, X_{4}\right]=0$. Thus, three generators $X_{1}, X_{2}$ and $X_{3}$ form the $s u(1,1)$ subalgebra, whereas the generator $X_{4}$ defines the commuting subalgebra $u(1)$.

All the CS transformations can be realized linearly in the following way. Let $\mathcal{Z}=$ $\left(\mathcal{Z}_{1}, \mathcal{Z}_{2}\right)$ be the complex $2 \times 1$ row whose finite transformation is:

$$
\mathcal{Z} \rightarrow \mathcal{G}^{\text {left }} \mathcal{Z G}^{\text {right }}
$$

We define $\mathcal{G}^{\text {left }}$ as the $U(1)$ group factor, and $\mathcal{G}^{\text {right }}$ as the $S U(1,1)$ group matrix:

$$
\mathcal{G}^{\text {left }} \overline{\mathcal{G}}^{\text {left }}=1, \quad \mathcal{G}^{\text {right }} \sigma_{3}\left(\mathcal{G}^{\text {right }}\right)^{+}=\sigma_{3}
$$

Then in the infinitesimal case $\mathcal{G}^{\text {left }}=1+i \gamma^{4}$ and $\mathcal{G}^{\text {right }}=1+\sum \gamma^{i} \tau_{i}$, where the $S U(1,1)$ matrix generators are:

$$
\tau_{1}=\frac{1}{2} \sigma_{1}=\frac{1}{2}\left(\begin{array}{ll}
0 & 1 \\
1 & 0
\end{array}\right), \quad \tau_{2}=\frac{i}{2} \sigma_{2}=\frac{i}{2}\left(\begin{array}{cc}
0 & -1 \\
1 & 0
\end{array}\right), \quad \tau_{3}=-\frac{i}{2} \sigma_{3}=-\frac{i}{2}\left(\begin{array}{cc}
1 & 0 \\
0 & -1
\end{array}\right) .
$$

The corresponding functional form of the generators is:

$$
\begin{aligned}
Y_{1} & =\frac{1}{2}\left(Z_{2} \partial_{1}+Z_{1} \partial_{2}\right), & Y_{2} & =\frac{i}{2}\left(Z_{2} \partial_{1}-Z_{1} \partial_{2}\right), \\
Y_{3} & =\frac{i}{2}\left(Z_{2} \partial_{2}-Z_{1} \partial_{1}\right), & Y_{4} & =\frac{i}{2}\left(Z_{1} \partial_{1}+Z_{2} \partial_{2}\right),
\end{aligned}
$$

where $Y_{4}$ is the generator for $\mathcal{G}^{\text {left }}$. One can prove that they satisfy the same commutation relations as $X$ 's.

Now we identify the $X$-generators and the $Y$-generators with the equal subscripts. Supposing that the functional relations $E=E\left(\mathcal{Z}_{1}, \mathcal{Z}_{2}\right)$ and $F=F\left(\mathcal{Z}_{1}, \mathcal{Z}_{2}\right)$ exist, we obtain the differential equations of the first order which define them. Our plan is following: we will consider the differential equations arising from the two identifications $Y_{1} \pm i Y_{2}=X_{1} \pm i Y_{2}$. Then $Y_{3}=X_{3}$ will take place in view of the $s u(1,1)$-algebra commutation relations, and the last equality $Y_{4}=X_{4}$ will be satisfied because we have normalized these generators in a 'right' way (in the other case they will be proportional). 
Let us briefly consider the solution process. The ' \pm '-identifications have the form:

$$
\begin{aligned}
& \sqrt{2} \mathcal{Z}_{1} \partial_{2}=(E+1) \partial_{F}, \\
& \sqrt{2} \mathcal{Z}_{2} \partial_{1}=-F(E-1) \partial_{E}-F^{2} \partial_{F} .
\end{aligned}
$$

Eq. (A.9) is equal to

$$
E_{, 2}=0, \quad \sqrt{2} \mathcal{Z}_{1} F_{, 2}=E+1 .
$$

From this we conclude that $E=E\left(\mathcal{Z}_{1}\right)$ and $F=\frac{1}{\sqrt{2}} \frac{\mathcal{Z}_{2}}{\mathcal{Z}_{1}}(E+1)+\psi\left(\mathcal{Z}_{1}\right)$, where $\psi$ is the arbitrary function. Next, Eq. (A.10) is consistent if $\psi=0$; the following integration leads to

$$
E=\frac{2}{C \mathcal{Z}_{1}-1}+1, \quad F=\sqrt{2} \frac{C \mathcal{Z}_{2}}{C \mathcal{Z}_{1}-1}
$$

where $C$ is the arbitrary constant. It is obvious that the linearizing potentials are defined at least up to multiplier. Then, using the replacement $C \mathcal{Z} \rightarrow \mathcal{Z}$, and inversing the formulae (A.12) we obtain that

$$
\mathcal{Z}_{1}=\frac{2}{E-1}+1, \quad \mathcal{Z}_{2}=\sqrt{2} \frac{F}{E-1}
$$

These potentials had been introduced at the first time tby Mazur in [22] without any relation to the CS subgroup linearization problem.

\section{Appendix B: CS Algebra for HS}

In this Appendix we compute the commutation relations for the charging symmetry algebra of the heterotic string theory with arbitrary $d$ and $n$.

First, the generators of the subgroup $\mathcal{G}^{\text {left }}$ form the $s o(2, d-1)$ algebra in the usual way. Let us consider the generators $\Gamma^{\text {right }}$ of the $s o(2, d-1+n)$ algebra (see Eq. (4.17)). They are constructed in the infinitesimal form; this means that $\lambda_{\mathcal{E}}=-2 \xi e, \lambda_{\mathcal{H}}=\sqrt{2} \xi h$ and $\tau=\xi t$, where $\xi$ is the infinithesimal parameter. The matrices $e, h$ and $t$ are finite $\left(e^{T}=-e\right.$, $t^{T}=-t$ ); they define the finite form of the NET, NHT and EMT generators:

$$
\Gamma_{N E T}(e)=\left(\begin{array}{cc}
\Sigma e & 0 \\
0 & 0
\end{array}\right), \quad \Gamma_{N H T}(h)=\left(\begin{array}{cc}
0 & \Sigma h \\
-h^{T} & 0
\end{array}\right), \quad \Gamma_{E M T}(t)=\left(\begin{array}{cc}
0 & 0 \\
0 & t
\end{array}\right) .
$$


The computation of the 'mixing' commutators gives:

$$
\begin{aligned}
& {\left[\Gamma_{E M T}(t), \Gamma_{N E T}(e)\right]=0} \\
& {\left[\Gamma_{N E T}(e), \Gamma_{N H T}(h)\right]=\Gamma_{N H T}(e \Sigma h),} \\
& {\left[\Gamma_{N H T}(h), \Gamma_{E M T}(t)\right]=\Gamma_{N H T}(h t) .}
\end{aligned}
$$

Next, for the 'inner' commutation relations one obtains:

$$
\begin{aligned}
& {\left[\Gamma_{E M T}\left(t_{1}\right), \Gamma_{E M T}\left(t_{2}\right)\right]=\Gamma_{E M T}\left(t_{[1} t_{2]}\right),} \\
& {\left[\Gamma_{N E T}\left(e_{1}\right), \Gamma_{N E T}\left(e_{2}\right)\right]=\Gamma_{N E T}\left(e_{[1} \Sigma e_{2]}\right)} \\
& {\left[\Gamma_{N H T}\left(h_{1}\right), \Gamma_{N H T}\left(h_{2}\right)\right]=-\Gamma_{N E T}\left(h_{[1} h_{2]}^{T}\right)-\Gamma_{E M T}\left(h_{[1}^{T} \Sigma t_{2]}\right) .}
\end{aligned}
$$

Thus, the NET and EMT subalgebras commute, whereas the commutator of NHT with NET and EMT is NHT again. However, NHT does not form a subalgebra with NET or

EMT, because from the 'inner' commutation relations it follows that the minimal algebra including NHT is equal to the full 'right' sector of the charging symmetry algebra, i.e. to so $(2, d-1+n)$.

\section{References}

[1] J. Scherk and J. H. Schwarz. Nucl. Phys. B81 (1974) 118.

[2] E. Witten, Nucl. Phys. B443 (1995) 85.

[3] E. Kiritsis, Introduction to Superstring Theory, CERN-TH/97-218, hep-th/9709062.

[4] J. Maharana and J.H. Schwarz, Nucl. Phys. B390 (1993) 3.

[5] A. Sen, Nucl. Phys. B434 (1995) 179.

[6] C.M. Hull and P.K. Townsend, Nucl. Phys. B438 (1995) 109.

[7] W. Kinnersley, J.Math.Phys. 21 (1980) 2231.

[8] A. Herrera-Aguilar and O. Kechkin, Int. J. Mod. Phys. A13 (1998) 393.

[9] F. J. Ernst, Phys. Rev. 1675 (1968) 1175.

[10] I. Bakas, M. Bourdeau and G. L. Cardoso, Nucl. Phys. B510 (1998) 103. 
[11] A. Font, L.E. Ibanez, D. Lust and F. Quevedo, Phys. Lett. B249 (1990) 35.

[12] W. Kinnersley, J. Math. Phys. 18 (1977) 1529.

[13] A. Herrera-Aguilar and O. Kechkin, Mod. Phys. Lett. A13 (1998) 1907.

[14] D.V. Gal'tsov and O.V. Kechkin, Phys. Lett. B361 (1995) 52.

[15] A. Herrera-Aguilar and O. Kechkin, Israel-Wilson-Perjes Solutions in Heterotic String Theory, to appear in Int. J. Mod. Phys. A, hep-th/9806154; Mod. Phys. Lett. A13 (1998) 1979.

[16] M. Cvetic and D. Youm, Phys. Rev. Lett. (1995) 4165.

[17] T. Matos, G. Rodriguez and R. Becerril J. Math. Phys. 33 (1992) 3521.

[18] V.A. Belinskii and V.E. Zakharov, Sov.Phys.JETP 48 (1978) 985; V.A. Belinskii and R. Ruffini, Phys.Lett. B89 (1980) 195.

[19] G.W. Gibbons and C.M. Hull, Phys. Lett. B109 (1982) 190. K.P. Tod, Phys. Lett. B121 (1983) 241.

[20] E. Cremmer, J. Scherk and S. Ferrara, Nucl. Phys. B74 (1978) 61; E. Bergshoeff, R. Kallosh and T. Ortin, Nucl.Phys. B478 (1996) 156.

[21] P.A.M. Dirac, Proc. Roy. Soc. A33 (1931) 60; J. Schwinger, Science 165 (1969) 757; D. Zwanziger, Phys. Rev. 176 (1968) 1489.

[22] P.O. Mazur, Acta Phys. Pol. 14 (1983) 219. 